\title{
ACTG2 Variants in Pediatric Chronic Intestinal Pseudo-obstruction With Megacystis
}

\author{
Jong Woo Hahn, ${ }^{1}$ Soo Young Moon, ${ }^{1}$ Min Soo Kim, ${ }^{1}$ Min Hyung Woo, ${ }^{1}$ Min Ji Sohn, ${ }^{1}$ Hyun-Young Kim, ${ }^{2}$ Moon-Woo Seong, ${ }^{3}$ \\ Sung Sup Park, ${ }^{3}$ Sung-Hye Park, ${ }^{4}$ Jin Soo Moon, ${ }^{1}$ and Jae Sung Ko ${ }^{1 *}$ \\ Departments of ${ }^{1}$ Pediatrics, ${ }^{2}$ Surgery, ${ }^{3}$ Laboratory Medicine, and ${ }^{4}$ Pathology, Seoul National University College of Medicine, Seoul, Korea
}

\begin{abstract}
Background/Aims
Chronic intestinal pseudo-obstruction (CIPO) is a clinically heterogeneous syndrome characterized by compromised peristalsis and intestinal obstruction. Variants of actin gamma 2 (ACTG2), a protein crucial for correct enteric muscle contraction, have been found in CIPO patients. The aim of this study is to examine the clinical features and ACTG2 variants in Korean patients with CIPO.
\end{abstract}

\section{Methods}

From January 1995 to August 2020, 12 patients diagnosed with CIPO were included and genetic analysis testing of ACTG2 was performed.

\section{Results}

Heterozygous ACTG2 missense variants were found in 6 patients (50.0\%). The p.Arg257Cys variant was found in 3 patients, and p.Arg63GIn and p.Arg178His variants were found in 1 patient each. A novel variant, p.lle193Phe, was found in 1 patient. Three patients were diagnosed at birth, 2 at the age of 1 year, and 1 at 3 years of age. Abnormal prenatal genitourinary ultrasonographic findings were found in all 6 patients; microcolon was found in 4 patients (66.7\%), and megacystis in all 6 patients. The pathology showed abnormal ganglion cells as well as myopathic findings. All patients are dependent on total parenteral nutrition and are to date alive.

\section{Conclusions}

ACTG2 variants are commonly found in Korean patients with CIPO. In CIPO patients with megacystis and abnormal prenatal ultrasonography, genetic testing of ACTG2 should be considered. Molecular diagnosis of CIPO is more important than pathologic diagnosis.

(J Neurogastroenterol Motil 2022;28:104-110)

Key Words

ACTG2; Intestinal pseudo-obstruction; Megacystis; Visceral myopathy

Received: October 30, 2020 Revised: February 23, 2021 Accepted: March 8, 2021

() This is an Open Access article distributed under the terms of the Creative Commons Attribution Non-Commercial License (http://creativecommons. org/licenses/by-nc/4.0) which permits unrestricted non-commercial use, distribution, and reproduction in any medium, provided the original work is properly cited.

*Correspondence: Jae Sung Ko, MD, PhD

Division of Pediatric Gastroenterology, Hepatology and Nutrition, Department of Pediatrics, Seoul National University College of Medicine, Seoul National University Children's Hospital, 101 Daehak-ro, Jongno-gu, Seoul 03080, Korea

Tel: +82-2-2072-2197, Fax: +82-2-743-3455, E-mail: kojs@snu.ac.kr 


\section{Introduction}

Chronic intestinal pseudo-obstruction (CIPO) is a disease with a wide variety of clinical and histological features, characterized by a decrease in intestinal peristalsis and symptoms of intestinal obstruction. ${ }^{1}$ A nationwide survey in the United States reported that there were $100 \mathrm{CIPO}$ patients each year, and another nationwide survey in Japan reported an estimated pediatric prevalence of 3.7 per 1 million individuals. ${ }^{2,3} \mathrm{CIPO}$ can involve any segment of the gastrointestinal and genitourinary tracts, and gastrointestinal symptoms, such as abdominal distention, abdominal pain and vomiting, and urinary symptoms, such as urination disorder, may occur. ${ }^{1,4}$ Patients with CIPO often undergo ileostomy and colostomy repeatedly. ${ }^{4-7}$

CIPO is classified as either a myopathy or a neuropathy, depending on histopathology. The genetic basis of CIPO was first reported by Lehtonen et $\mathrm{al}^{8}$ in 2012 . Since then, several genes related to CIPO have been discovered, of which actin gamma 2 (ACTG2) is the most common. ${ }^{4,6,7,9}$ The ACTG2 is located in the short arm of chromosome 2 , encoding gamma 2 enteric actin, which is found in the smooth muscle cells of the urinary and intestinal tracts. ACTG2 is crucial for intestinal smooth muscle contraction, ${ }^{9,10}$ and variants of ACTG2 are associated with visceral myopathy and megacystismicrocolon-intestinal hypoperistalsis syndrome (MMIHS). ${ }^{6,10,11}$ However, there are no studies on ACTG2 variants in Asia.

The purpose of this study is to investigate the clinical features and ACTG2 variants in Korean patients with CIPO.

\section{Materials and Methods}

\section{Study Population and Data Collection}

In patients with suspected intestinal obstruction, abdominal radiography is performed to determine if there is bowel dilation and air-fluid levels. If there is no fixed lesion or mechanical obstruction, it can be diagnosed as a pseudo-obstruction. If the symptoms per- sist for more than 6 months or postnatally for more than 2 months, CIPO may be diagnosed. ${ }^{11} \mathrm{~A}$ total of 12 patients diagnosed with CIPO at National University Hospital from January 1995 to August 2020 were included. Twelve patients were examined, and their medical records were retrospectively analyzed. Medical records included age, sex, age at onset, presenting symptoms, imaging studies, laboratory findings, and growth curves.

This study was approved by the Institutional Review Board (No. 1910-065-1070) at the performing institution. IBM SPSS Statistics 25 software was used to complete the statistical analysis. Fisher's exact test was performed to compare the clinical features between patients with and without ACTG2 variants. A $P$-value $<$ 0.05 was considered statistically significant.

\section{Genetic Testing of ACTG2}

Among the 12 patients, 11 underwent direct sequencing of ACTG2 and 1 was tested for the CIPO gene panel. The CIPO gene panel contained the following genes: ACTA2, ACTG2, CLMP, FLNA, L1CAM, LMOD1, MYH11, MYLK, POLG, RAD21, SGOL1, SOX10, and TYMP. Genomic DNA from peripheral leukocytes was extracted. All 8 coding exons and flanking introns of ACTG2 were amplified using polymerase chain reaction. Genomic DNA targeted regions were sequenced using the Sanger sequencing system. The common variants were filtered using gnomAD (http://gnomad.broadinstitute.org) and KRG (http://coda.nih.go.kr/coda/KRGDB) databases. The Human Gene Mutation Database and ClinVar were used to search for known pathogenic variants. The sequence variants were evaluated by computational (in silico) predictive programs using PolyPhen-2, SIFT, and MutationTaster. The effect of sequence variants was determined at the nucleotide and amino acid levels, and the potential impact of the variants on the protein could be seen. In addition, the pathogenicity of sequence variants was evaluated using the 2015 American College of Medical Genetics ad Genomics (ACMG) guidelines. $^{12}$

Table 1. Variants of ACTG2 Gene Among Patients With Chronic Intestinal Pseudo-obstruction

\begin{tabular}{cccccc}
\hline DNA change & Amino acid change & Variant type & Allele frequency & & \multicolumn{2}{c}{ ACMG classification } \\
\hline $769 \mathrm{C}>\mathrm{T}$ & p.Arg257Cys & Missense & $3 / 6(50.0 \%)$ & PM1, PM2, PP2, PP3, PP5 & Likely pathogenic \\
$188 \mathrm{G}>\mathrm{A}$ & p.Arg63Gln & Missense & $1 / 6(16.7 \%)$ & PM1, PM2, PP2, PP3 & Likely pathogenic \\
$577 \mathrm{~A}>\mathrm{T}$ & p.Ile193Phe & Missense & $1 / 6(16.7 \%)$ & PM1, PM2, PP2, PP3 & Likely pathogenic \\
$533 \mathrm{G}>\mathrm{A}$ & p.Arg178His & Missense & $1 / 6(16.7 \%)$ & PM1, PM2, PP2, PP3, PP5 & Likely pathogenic \\
\hline
\end{tabular}

ACTG2, actin gamma 2; CIPO, chronic intestinal pseudo-obstruction; ACMG, American College of Medical Genetics and Genomics; PM, pathogenic moderate; $\mathrm{PP}$, pathogenic supporting. 


\section{Results}

\section{ACTG2 Variant Analysis}

Twelve patients were analyzed, and 6 were identified with ACTG2 variants. All 6 cases were sporadic and without a family history. All variants were heterozygous missense variants, of which 5 were arginine substitutions. The variants c.769C > T, p.Arg257Cys (50.0\%) was found in 3 patients; c.188G >A, p.Arg63Gln (16.7\%) in 1 patient; c. $533 \mathrm{G}>\mathrm{A}$, p.Arg $178 \mathrm{His}(16.7 \%)$ in 1 patient; and c.577A $>$ T, p.Ile193Phe $(16.7 \%)$ in 1 patient (Table 1 ). p.Arg257Cys, p.Arg178His, and p.Arg63Gln are previously reported variants and p.Arg257Cys is the most common. p.Ile193Phe is a novel variant that is not observed among the normal population and is located at highly conserved loci in various species. All variants were considered likely pathogenic by the ACMG classification. $^{12}$

\section{Comparison of Clinical Features Between Patients With and Without ACTG2 Variants}

Table 2 compares patients with and without ACTG2 variants. The presence of megacystis and abnormal prenatal ultrasonography showed a statistically significant difference between the 2 groups, but others, including age of diagnosis, microcolon, malrotation, hydronephrosis, neurogenic bladder, long-term clean intermittent catheterization (CIC), and long-term home parenteral nutrition $(\mathrm{PN})$, were not statistically significant.

\section{Clinical Features of Patients With ACTG2 Variants}

The clinical characteristics of the 6 patients with ACTG2 vari-

Table 2. Comparison of Clinical Feature Between Patients With and Without ACTG2 Mutations

\begin{tabular}{lllc}
\hline \multicolumn{1}{c}{ Clinical feature } & \multicolumn{1}{c}{$\begin{array}{c}\text { ACTG2 }(+) \\
(\mathrm{n}=6)\end{array}$} & $\begin{array}{l}\text { ACTG2(-) } \\
(\mathrm{n}=6)\end{array}$ & $P$-value \\
\hline Age at diagnosis & 7 months & Birth & $>0.05$ \\
Prenatal ultrasonography & $6 / 6(100 \%)$ & $1 / 6(16.7 \%)$ & 0.015 \\
Microcolon & $4 / 6(66.7 \%)$ & $0 / 6(0.0 \%)$ & 0.061 \\
Malrotation & $3 / 6(50 \%)$ & $0 / 6(0.0 \%)$ & $>0.05$ \\
Megacystis & $6 / 6(100 \%)$ & $0 / 6(0.0 \%)$ & 0.002 \\
Hydronephrosis & $4 / 6(66.7 \%)$ & $1 / 6(16.7 \%)$ & $>0.05$ \\
Neurogenic bladder & $2 / 6(33.3 \%)$ & $0 / 6(0.0 \%)$ & $>0.05$ \\
Long-term CIC & $2 / 6(33.3 \%)$ & $0 / 6(0.0 \%)$ & $>0.05$ \\
Long-term home PN & $6 / 6(100 \%)$ & $6 / 6(100.0 \%)$ & $>0.05$ \\
\hline
\end{tabular}

$\mathrm{CIC}$, clean intermittent catheterization; $\mathrm{PN}$, parenteral nutrition. ants are summarized in Table 3. Four patients were female, and 2 were male. Their current ages range from 44 months to 27 years with a median value of 8.5 years. Three patients were diagnosed at birth: 2 at age 1 year, and 1 at age 3 years, with the median age at diagnosis being 7 months. The initial clinical symptoms were constipation in 5 patients, abdominal distention in 3 , and vomiting in 2. On prenatal ultrasonography, all patients showed abnormal genitourinary findings, such as enlarged bladder, ovarian cyst, and hydronephrosis. Megacystis was observed in all 6 patients. Microcolon was found in 4 patients (66.7\%), and they were diagnosed with MMIHS. Malrotation was observed in 3 patients (50.0\%), hydronephrosis in 4 (66.7\%), and neurogenic bladder in 2 (33.3\%). Pathological studies in all patients showed abnormalities of the muscle layer and ganglion cells. Thinning of the muscle layer and vacuolar changes of the muscle layer were found in 3 patients each. Nuclear palisading and acidophilic globular bodies were found in 1 patient each (Figure). All patients had hypoganglionosis and immature ganglion cells, and ganglion cell shapes were smaller than normal.

\section{Long-term Outcomes in Patients With ACTG2 Variants}

The follow-up period of patients ranged from 44 months to 24 years with a median value of 62 months. All patients underwent abdominal surgeries. Jejunostomy was performed in 2 patients, ileostomy in all 6 patients, and colostomy in 1 patient. Cholecystectomy, gastrectomy, and colectomy were performed in Case 3. None of the patients received an intestinal transplant and all patients are still alive. Long-term CIC was performed in 2 patients (33.3\%), and Case 6 underwent vesicotomy 5 months immediately after diagnosis. All patients remain dependent on PN with oral feeding. Among them, Case 4 recently discontinued PN, whereas Case 5 recently initiated PN. Central line-associated bloodstream infection (CLABSI) was observed in 3 patients, and fatty liver was observed in 2 patients. One patient suffered recurrent small intestinal bacterial overgrowth and currently takes cyclic antibiotics. Pyridostigmine, an acetylcholinesterase inhibitor that stimulates gastrointestinal motility, was administered to 3 patients, and symptoms improved in 2 of them.

\section{Discussion}

In this study, we investigated the spectrum of clinical features and ACTG2 variants in Korean patients with CIPO. Among the 12 CIPO patients, $A C T G 2$ variants were found in 6 patients 


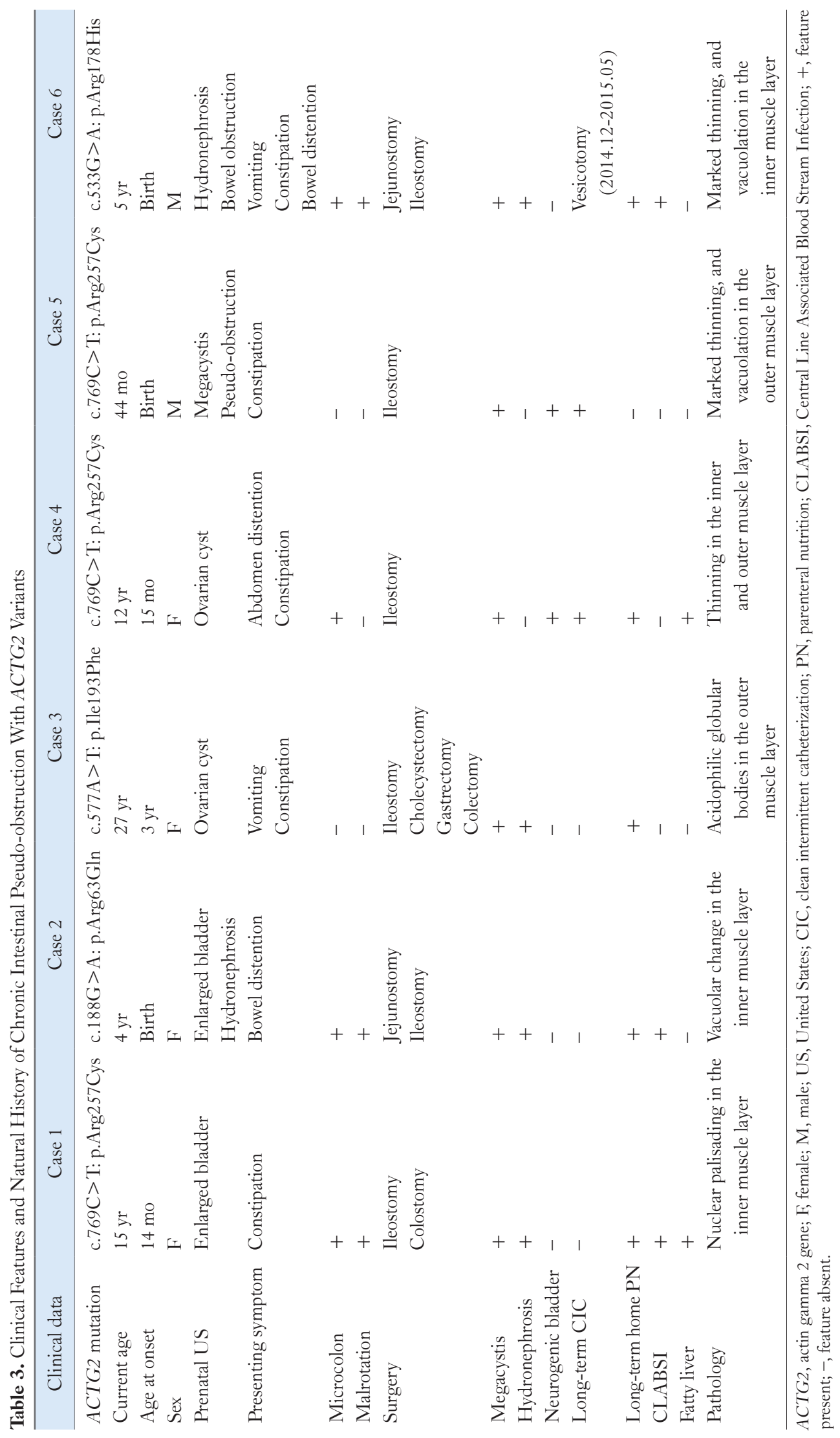



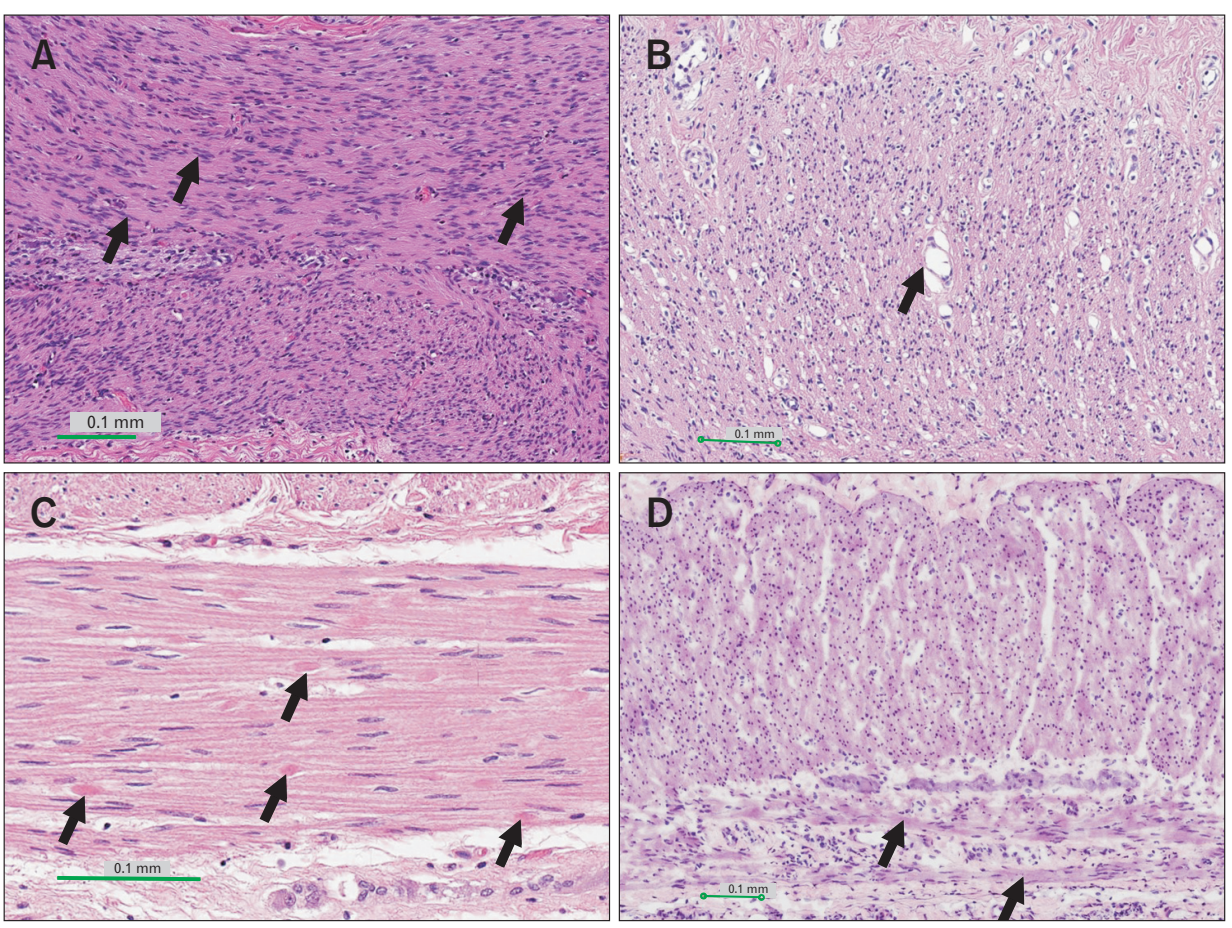

Figure. Pathologic findings of patients with ACTG2 variants. (A) [Case 1] The inner circular muscle layer shows nuclear palisading (arrows). (B) [Case 2] The inner circular muscle layer shows vacuolar change (arrow). (C) [Case 3] The outer layer of the muscle shows acidophilic globular bodies (arrows). (D) [Case 5] The outer muscle layer shows marked thinning (arrows) $(\mathrm{H} \& \mathrm{E}, \mathrm{bar}=0.1 \mathrm{~mm})$.
(50.0\%), with p.Arg257Cys being the most common. All cases were sporadic, and we reported a novel ACTG2 variant. All patients with ACTG2 variants showed megacystis, abnormal prenatal ultrasonography, and had pathological abnormalities in the muscle layer and ganglion cells. All patients were dependent on $\mathrm{PN}$ and are alive to date. We showed the indication and importance of ACTG2 genetic testing in patients with CIPO.

In this study, 4 ACTG2 variants were identified in 6 children, and 3 of the 4 variants had arginine substitutions. In previous studies, $44.0-62.0 \%$ of CIPO patients were positive for ACTG2 variants, and arginine substitutions accounted for $49.0 \%$ of total variants. ${ }^{13,14}$ All variants in this study are heterozygous missense variants, which is consistent with findings of other studies. ${ }^{15}$ p.Arg257Cys, p.Arg63Gln, and p.Arg178His have has been previously reported and are recurrent pathogenic variants, ${ }^{7,10}$ while p.Ile193Phe is novel. All variants including a novel variant were considered likely pathogenic by the ACMG classification. These known missense variants are reported to affect protein function, impair ACTG2 polymerization, and reduce smooth muscle cell contractility. ${ }^{15}$ Previous studies have shown that arginine substitution at several different sites affects the function of actin filament, contributing to poor contractility of smooth muscle in COS7, CRL-1776 and U2OS cells. ${ }^{8,9}$ In this study, p.Arg257Cys was the most common variant, and p.Arg178Cys and p.Arg257Cys were mutation hot spots in other studies. ${ }^{6,10,13}$ All patients in this study were sporadic, and these variants may have occurred de novo. Cases 1,4 , and 5 had the same variant as p.Arg257Cys, but the clinical features were different. Cases 1 and 4 were diagnosed with MMIHS. However, Case 5 did not have MMIHS because microcolon was not present. In previous studies, microcolon was reported in 5 out of 20 (25.0\%) patients with p.Arg257Cys. ${ }^{13}$ These findings suggest that visceral myopathy and MMIHS are one entity with a continuum of various symptoms. Some studies have shown a genotype-phenotype correlation. Arginine missense substitutions in ACTG2 had a $63.8 \%$ chance of requiring intestinal transplant, total PN dependence, or disease-related mortality. ${ }^{13}$ In this study, all patients were dependent on total $\mathrm{PN}$, regardless of arginine substitutions. In addition, all variants in this study may occur de novo, which may present with a more severe phenotype than when inherited. ${ }^{6}$ All patients in this study showed a severe phenotype, and the number of patients was small; therefore, it was difficult to evaluate a genotype-phenotype correlation. Also, there was no racial difference between Asian and Caucasian patients in ACTG2 variants, because p.Arg257Cys, frequently found in Caucasians, was the most common in this study. ${ }^{6,710}$ Out of 12 patients, 1 patient was examined with the CIPO gene panel and an ACTG2 variant was identified.

We found that in the patient group with ACTG2 variants, there were 4 female and 2 male patients, which is consistent with previous studies showing a female preponderance. ${ }^{13,16}$ In this study, megacystis was found in all 6 patients. In previous studies, 85.0- 
$100.0 \%$ of patients with ACTG2 variants had megacystis. ${ }^{6,13} \mathrm{On}$ prenatal ultrasonography, all patients with ACTG2 variants had genitourinary abnormalities. All MMIHS patients with ACTG2 variants had prenatally evident fetal megacystis. ${ }^{17}$ Taken together, if patients with CIPO have genitourinary abnormalities on prenatal ultrasonography or presence of megacystis, the evaluation of visceral myopathy with ACTG2 variants should be considered.

Thinning and vacuolar changes of the muscle layer found in this study are key features of visceral myopathy. Nuclear palisading is a nonspecific histopathologic findings of myopathy, which is occasionally observed in other studies. ${ }^{18,19}$ Acidophilic globular body is a novel and pathologic finding, which is not observed in normal intestinal muscle proper, and appears to be related with muscular degenerative changes. Also, ganglion cell abnormalities such as hypoganglionosis and immature ganglia were found in all 6 patients with ACTG2 variants. In previous studies, ganglion cell abnormalities were observed in patients with visceral myopathy or MMIHS. ${ }^{210,17,20-24}$ Ganglion cell abnormalities may be secondary changes in myopathy due to ACTG2 variants. ${ }^{10}$ Because hypoganglionosis or dysmorphic ganglion cells may be seen in CIPO with ACTG2 variants, it is difficult to diagnose by histopathology alone, and molecular diagnosis is more accurate than histologic diagnosis.

In this study, different abdominal surgeries were performed. Six patients received 26 surgeries and a mean of 4.3 surgeries per patient, with the most common operation being ostomy, which is similar to the results of other studies. ${ }^{7,13,22,25}$ In this study, all patients were dependent on $\mathrm{PN}$, which is similar to the results of other studies. ${ }^{13,25,26}$ Long-term home PN causes several complications. ${ }^{27,28}$ CLABSI was observed in 3 patients (50.0\%), and fatty liver was observed in 2 patients $(33.3 \%)$ in this study. To reduce the complications of long-term $\mathrm{PN}$, fish oil-based lipid emulsions are used and enteral feeding is maintained. ${ }^{11,16}$ Thus, cirrhosis may not develop in this study. Previous studies have reported that the survival rate was 12.6-19.7\%, and the oldest survivor was 25 years old. ${ }^{7,16,22}$ Mortality was associated with a short small intestine, involvement of the urinary system, $<1$ year of age of onset, and myopathy on histology. ${ }^{29}$ In this study, the survival rate of CIPO was $100.0 \%$, and the oldest survivor was 27 years old. The remarkable improvement in prognosis in this study may have resulted from improvements in nutritional management and supportive care with intestinal rehabilitation programs. ${ }^{7,26,30,31}$ In this study, pyridostigmine was administered to 3 patients, and was effective in 2 of them. Pyridostigmine has been demonstrated to be effective in colonic decompression in pediatric CIPO patients. ${ }^{32,33}$

The limitation of this study is that the number of CIPO pa- tients was small, and the study was conducted at a single center. A multicenter study involving a large number of Korean patients with CIPO is needed.

In conclusion, ACTG2 variants are commonly found among CIPO patients in Korea. In CIPO patients with megacystis and abnormal prenatal ultrasonography, genetic testing of ACTG2 should be considered. Molecular diagnosis of CIPO is important because it is difficult to differentiate myopathy from neuropathy by pathological findings alone. ACTG2 genetic analysis can help avoid invasive diagnostic interventions such as full-thickness bowel biopsy.

Financial support: This study was supported by grant (No. 0420203090) from Seoul National University Hospital.

\section{Conflicts of interest: None.}

Author contributions: Jong Woo Hahn: collecting, interpreting data, and drafting the manuscript; Soo Young Moon, Min Soo Kim, Min Hyung Woo, and Min Ji Sohn: interpreting data; Hyun-Young Kim: conducting the study; Moon-Woo Seong, Sung Sup Park, and Sung-Hye Park: conducting the study and interpreting data; Jin Soo Moon: conducting the study, interpreting the data, and revising the manuscript; and Jae Sung Ko: planning and conducting the study, interpreting the data, and revising the manuscript.

\section{References}

1. Collins RRJ, Barth B, Megison S, et al. ACTG2-associated visceral myopathy with chronic intestinal pseudoobstruction, intestinal malrotation, hypertrophic pyloric stenosis, choledochal cyst, and a novel missense mutation. Int J Surg Pathol 2019;27:77-83.

2. Muto M, Matsufuji H, Tomomasa T, et al. Pediatric chronic intestinal pseudo-obstruction is a rare, serious, and intractable disease: a report of a nationwide survey in Japan. J Pediatr Surg 2014;49:1799-1803.

3. Di Lorenzo C. Pseudo-obstruction: current approaches. Gastroenterology 1999;116:980-987.

4. Di Nardo G, Di Lorenzo C, Lauro A, et al. Chronic intestinal pseudoobstruction in children and adults: diagnosis and therapeutic options. Neurogastroenterol Motil 2017;29:e12945.

5. Yamataka A, Ohshiro K, Kobayashi H, et al. Abnormal distribution of intestinal pacemaker (C-KIT-positive) cells in an infant with chronic idiopathic intestinal pseudoobstruction. J Pediatr Surg 1998;33:859-862.

6. Milunsky A, Baldwin C, Zhang X, Primack D, Curnow A, Milunsky J. Diagnosis of chronic intestinal pseudo-obstruction and megacystis by sequencing the ACTG2 gene. J Pediatr Gastroenterol Nutr 2017;65:384387.

7. Wangler MF, Gonzaga-Jauregui C, Gambin T, et al. Heterozygous de novo and inherited mutations in the smooth muscle actin (ACTG2) 
gene underlie megacystis-microcolon-intestinal hypoperistalsis syndrome. PLoS Genet 2014;10:e1004258.

8. Lehtonen HJ, Sipponen T, Tojkander S, et al. Segregation of a missense variant in enteric smooth muscle actin gamma- 2 with autosomal dominant familial visceral myopathy. Gastroenterology 2012;143:1482-1491, e3.

9. Thorson W, Diaz-Horta O, Foster J 2nd, et al. De novo ACTG2 mutations cause congenital distended bladder, microcolon, and intestinal hypoperistalsis. Hum Genet 2014;133:737-742.

10. Matera I, Rusmini M, Guo Y, et al. Variants of the ACTG2 gene correlate with degree of severity and presence of megacystis in chronic intestinal pseudo-obstruction. Eur J Hum Genet 2016;24:1211-1215.

11. Rudolph CD, Hyman PE, Altschuler SM, et al. Diagnosis and treatment of chronic intestinal pseudo-obstruction in children: report of consensus workshop. J Pediatr Gastroenterol Nutr 1997;24:102-112.

12. Richards S, Aziz N, Bale S, et al. Standards and guidelines for the interpretation of sequence variants: a joint consensus recommendation of the American College of Medical Genetics and Genomics and the Association for Molecular Pathology. Genet Met 2015;17:405-424.

13. Assia Batzir N, Kishor Bhagwat P, Larson A, et al. Recurrent arginine substitutions in the ACTG2 gene are the primary driver of disease burden and severity in visceral myopathy. Hum Mutat 2020;41:641-654.

14. Milunsky A, Lazier J, Baldwin C, Young C, Primack D, Milunsky JM. Prenatal diagnosis of chronic intestinal pseudo-obstruction and paternal somatic mosaicism for the ACTG2 pathogenic variant. Prenat Diagn 2017;37:1254-1256.

15. Halim D, Hofstra RM, Signorile L, et al. ACTG2 variants impair actin polymerization in sporadic megacystis microcolon intestinal hypoperistalsis syndrome. Hum Mol Genet 2016;25:571-583.

16. Gosemann JH, Puri P. Megacystis microcolon intestinal hypoperistalsis syndrome: systematic review of outcome. Pediatr Surg Int 2011;27:10411046.

17. Tuzovic L, Tang S, Miller RS, et al. New insights into the genetics of fetal megacystis: ACTG2 mutations, encoding gamma-2 smooth muscle actin in megacystis microcolon intestinal hypoperistalsis syndrome (berdon syndrome). Fetal Diagn Ther 2015;38:296-306.

18. Miyata M, Watanabe K, Suzuki T, et al. Chronic intestinal pseudoobstruction ileus in a patient with rheumatoid arthritis: outcome of a stoma construction and its histopathological features. J Gastroenterol 2002;37:653-657.

19. Rohrmann CA Jr, Ricci MT, Krishnamurthy S, Schuffler MD. Radiologic and histologic differentiation of neuromuscular disorders of the gastrointestinal tract: visceral myopathies, visceral neuropathies, and progressive systemic sclerosis. AJR Am J Roentgenol 1984;143:933-941.
20. Puri P, Lake BD, Gorman F, O’Donnell B, Nixon HH. Megacystismicrocolon-intestinal hypoperistalsis syndrome: a visceral myopathy. J Pediatr Surg 1983;18:64-69.

21. Rolle U, O'Briain S, Pearl RH, Puri P. Megacystis-microcolon-intestinal hypoperistalsis syndrome: evidence of intestinal myopathy. Pediatr Surg Int 2002;18:2-5.

22. Puri P, Shinkai M. Megacystis microcolon intestinal hypoperistalsis syndrome. Semin Pediatr Surg 2005;14:58-63.

23. Kirtane J, Talwalker V, Dastur DK. Megacystis, microcolon, intestinal hypoperistalsis syndrome: possible pathogenesis. J Pediatr Surg 1984;19:206-208.

24. Piotrowska AP, Rolle U, Chertin B, De Caluwé D, Bianchi A, Puri P. Alterations in smooth muscle contractile and cytoskeleton proteins and interstitial cells of Cajal in megacystis microcolon intestinal hypoperistalsis syndrome. J Pediatr Surg 2003;38:749-755.

25. Faure C, Goulet O, Ategbo S, et al. Chronic intestinal pseudoobstruction syndrome: clinical analysis, outcome, and prognosis in 105 children. French-speaking group of pediatric gastroenterology. Dig Dis Sci 1999;44:953-959.

26. Stanghellini V, Cogliandro RF, De Giorgio R, et al. Natural history of chronic idiopathic intestinal pseudo-obstruction in adults: a single center study. Clin Gastroenterol Hepatol 2005;3:449-458.

27. Knafelz D, Gambarara M, Diamanti A, et al. Complications of home parenteral nutrition in a large pediatric series. Transplant Proc 2003;35:3050-3051.

28. Diamanti A, Gambarara M, Knafelz D, et al. Prevalence of liver complications in pediatric patients on home parenteral nutrition: indications for intestinal or combined liver-intestinal transplantation. Transplant Proc 2003;35:3047-3049.

29. Cucchiara S, Borrelli O. Nutritional challenge in pseudo-obstruction: the bridge between motility and nutrition. J Pediatr Gastroenterol Nutr 2009;48(suppl 2):S83-S85.

30. Lapointe SP, Rivet C, Goulet O, Fékété CN, Lortat-Jacob S. Urological manifestations associated with chronic intestinal pseudo-obstructions in children. J Urol 2002;168(4 pt 2):1768-1770.

31. Fullerton BS, Hong CR, Jaksic T. Long-term outcomes of pediatric intestinal failure. Semin Pediatr Surg 2017;26:328-335.

32. Choudhury A, Rahyead A, Kammermeier J, Mutalib M. The use of pyridostigmine in a child with chronic intestinal pseudo-obstruction. Pediatrics 2018;141(suppl 5):S404-S407.

33. Lee H, Park S, Oh JT, Kim HM, Kim S, Lee JS. Oral pyridostigmineresponsive visceral myopathy with ACTG2 mutations: a case series. J Pediatr Gastroenterol Nutr 2019;68:e16-e17. 